\title{
Some ring theory from Jenô Szigeti
}

\author{
Stephan Foldes
}




\title{
SOME RING THEORY FROM JENŐ SZIGETI
}

\author{
STEPHAN FOLDES
}

Received 10 August, 2015

\begin{abstract}
A selection of ring theory papers by Jenő Szigeti is reviewed with an emphasis on aspects related to matrix algebras.
\end{abstract}

The present overview concentrates on three areas of Szigeti's work. "Eulerian polynomial identities" deals essentially with polynomials in several non-commuting indeterminates corresponding to directed Eulerian graphs. "Lie nilpotent determinant theory" adapts to the non-commutative case the classical concepts of determinant, adjoint and characteristic polynomial to yield analogues of well known linear algebra results, especially over Lie nilpotent rings. "Centralizers and zero-level centralizers" is about some non-commutative extensions of theorems on centralizers and double centralizers in matrix algebras, with additional considerations of two-sided annihilators.

We aim at a condensed but self contained presentation of a selection of results.

\section{EULERIAN POLYNOMIAL IDENTITIES}

Let $\Gamma$ be a directed graph with vertex set $V(\Gamma)=\{1,2, \ldots, k\}$ and edge set $E(\Gamma)=\left\{x_{1}, x_{2}, \ldots, x_{N}\right\}$. For each $1 \leq r \leq N, x_{r}$ is an edge from $\sigma(r)$ to $\tau(r)$, both in $V(\Gamma)$. A directed Eulerian path which starts at $p$ and ends at $q$ is viewed as a permutation $\pi$ of the edges (or rather their indices). The polynomial $P_{\Gamma}(X)$ in the set $X=E(\Gamma)$ of non-commuting indeterminates, induced by $\Gamma$, is defined as follows:

$$
P_{\Gamma}(X)=\sum_{\pi \in \Pi(\Gamma, p, q)} \operatorname{sgn}(\pi) x_{\pi(1)} x_{\pi(2)} \ldots x_{\pi(N)},
$$

where $\Pi(\Gamma, p, q)$ is the set of all directed Eulerian paths of $\Gamma$ from $p$ to $q$. The main result of [16] states that if $N \geq 2 k n$, then $P_{\Gamma}(X)=0$ is a polynomial identity on the $n \times n$ matrix ring $\mathrm{M}_{n}(\Omega)$, where $\Omega$ is an arbitrary commutative ring with 1 . This is a broad generalization of the famous Amitsur-Levitzki theorem. Surprisingly, the above graph based construction led to essentially new identities even for $3 \times 3$

Dedicated to the occasion of Jenő Szigeti's 60th birthday. 
matrices. The Capelli polynomials in [4] appear as Eulerian polynomials corresponding to a particular class of graphs, where the authors proved that the inequalities conditioning the corresponding matrix identities are sharp.

Assuming certain lower bounds on out degrees the sign free (permanental) version

$$
Q_{\Gamma}(X)=\sum_{\pi \in \Pi(\Gamma, p, q)} x_{\pi(1)} x_{\pi(2)} \ldots x_{\pi(N)}
$$

of $P_{\Gamma}(X)$ also yields the identity $Q_{\Gamma}(X)=0$ on $\mathrm{M}_{n}(\Omega)$, where $\Omega$ is a commutative ring of characteristic $d$. The lower bounds assumed depend on both $d$ and $n$ (see [7]). A variation on these assumptions on the graph $\Gamma$ which ensure the validity of the identity $Q_{\Gamma}(X)=0$ on $\mathrm{M}_{n}(\Omega)$ can be found in [2].

It is worth to note that the proof of the matrix identity $P_{\Gamma}(X)=0$ is based on the use of Swan's theorem on the signs of directed Eulerian paths, while the proof of $Q_{\Gamma}(X)=0$ is based on a formula (due to Aardenne-Ehrenfest and de Bruijn) counting the number of directed Eulerian paths. A particularly concise form of the mentioned theorem of Swan is formulated in [3] using a special adjacency matrix over the Grassmann algebra.

The form of the polynomial $P_{\Gamma}(X)$ is enriched in [8] by an involution operator * applied to indeterminates traversed in opposite direction in an undirected Eulerian path, providing identities involving matrix transposition also.

\section{LIE NILPOTENT DETERMINANT THEORY}

The following are the outlines of a new determinant theory developed in the series of papers $[5,6,9-12,14,15,18,19]$.

For an $n \times n$ matrix $A=\left[a_{i, j}\right]$ over a not necessarily commutative ring (or $K$ algebra) $R$ with 1 , the element

$$
\operatorname{sdet}(A)=\sum_{\alpha, \beta \in \mathrm{S}_{n}} \operatorname{sgn}(\alpha) \operatorname{sgn}(\beta) a_{\alpha(1), \beta(1)} \ldots a_{\alpha(t), \beta(t)} \ldots a_{\alpha(n), \beta(n)}
$$

of $R$ is called the symmetric determinant of $A$. The $(r, s)$ entry of the symmetric adjoint $A^{*}=\left[a_{i, j}^{*}\right]$ of $A$ is the signed symmetric determinant $a_{r, s}^{*}=(-1)^{r+s} \operatorname{sdet}\left(A_{s, r}\right)$ of the $(n-1) \times(n-1)$ minor $A_{s, r}$ of $A$ arising from the deletion of the $s$-th row and the $r$-th column of $A$. If $R$ is commutative, then $\operatorname{sdet}(A)=n ! \operatorname{det}(A)$ and $A^{*}=$ $(n-1) ! \operatorname{adj}(A), w h e r e \operatorname{det}(A)$ and $\operatorname{adj}(A)$ denote the ordinary determinant and adjoint of $A$.

The right adjoint sequence $\left(P_{k}\right)_{k \geq 1}$ of $A$ is defined by the following recursion: $P_{1}=A^{*}$ and $P_{k+1}=\left(A P_{1} \cdots P_{k}\right)^{*}$ for $k \geq 1$. The $k$-th right determinant of $A$ is the trace

$$
\operatorname{rdet}_{(k)}(A)=\operatorname{tr}\left(A P_{1} \cdots P_{k}\right)
$$

and the $k$-th right adjoint of $A$ is the product

$$
\operatorname{radj}_{(k)}(A)=n P_{1} \cdots P_{k} .
$$


An important observation is that

$$
\operatorname{rdet}_{(1)}(A)=\operatorname{tr}\left(A A^{*}\right)=\operatorname{sdet}(A)=\operatorname{tr}\left(A^{*} A\right) .
$$

For $2 \times 2$ and $3 \times 3$ matrices we have the following symmetric analogues of the Newton trace formulae : if $A \in \mathrm{M}_{2}(R)$, then

$$
\operatorname{sdet}(A)=\operatorname{tr}^{2}(A)-\operatorname{tr}\left(A^{2}\right)
$$

and if $A \in \mathrm{M}_{3}(R)$, then

$$
\begin{aligned}
\operatorname{sdet}(A)= & \operatorname{tr}^{3}(A)-\left[\operatorname{tr}(A) \cdot \operatorname{tr}\left(A^{2}\right)+\operatorname{tr}(A \cdot \operatorname{tr}(A) \cdot A)+\operatorname{tr}\left(A^{2}\right) \cdot \operatorname{tr}(A)\right] \\
& +\left[\operatorname{tr}\left(A^{3}\right)+\operatorname{tr}\left(\left(A^{\top}\right)^{3}\right)\right],
\end{aligned}
$$

where $A^{\top}$ denotes the transpose of $A$. The term preceded by the negative sign can also be expressed more symmetrically, with the notation $\operatorname{tr}(A)=a$, as the trace of $a A A+A a A+A A a$.

The $k$-th right characteristic polynomial of $A$ is the $k$-th right determinant of the $n \times n$ matrix $x I_{n}-A$ in $\mathrm{M}_{n}(R[x])$ :

$$
p_{A, k}(x)=\operatorname{rdet}_{(k)}\left(x I_{n}-A\right)
$$

and $p_{A, k}(x)$ is of the form

$$
p_{A, k}(x)=\lambda_{0}^{(k)}+\lambda_{1}^{(k)} x+\cdots+\lambda_{n^{k}-1}^{(k)} x^{n^{k}-1}+\lambda_{n^{k}}^{(k)} x^{n^{k}},
$$

where $\lambda_{0}^{(k)}, \lambda_{1}^{(k)}, \ldots, \lambda_{n^{k}-1}^{(k)}, \lambda_{n^{k}}^{(k)} \in R$ and $\lambda_{n^{k}}^{(k)}=n\{(n-1) !\}^{1+n+n^{2}+\cdots+n^{k-1}}$. Under additional assumptions on $A$ it is proved in a recent work [14] that the coefficients of $p_{A, k}(x)$ are in the fixed ring of a certain group of automorphisms of $R$.

If $R$ satisfies the commutator identity

$$
\left[\left[\left[\ldots\left[\left[x_{1}, x_{2}\right], x_{3}\right], \ldots\right], x_{k}\right], x_{k+1}\right]=0
$$

(i.e. if $R$ is Lie nilpotent of index $k$ ), then the product $A \cdot \operatorname{radj}_{(k)}(A)$ is a scalar matrix

$$
A \cdot \operatorname{radj}_{(k)}(A)=n A P_{1} \cdots P_{k}=\operatorname{rdet}_{(k)}(A) \cdot I_{n}
$$

and the Cayley-Hamilton identity with right scalar coefficients

$$
\text { (A) } p_{A, k}=I_{n} \lambda_{0}^{(k)}+A \lambda_{1}^{(k)}+\cdots+A^{n^{k}-1} \lambda_{n^{k}-1}^{(k)}+A^{n^{k}} \lambda_{n^{k}}^{(k)}=0
$$

holds for $A$. We also have $(A) u=0$, where $u(x)=p_{A, k}(x) h(x)$ and $h(x) \in R[x]$ is arbitrary. Clearly, these results can be viewed as the index $k$ Lie nilpotent version of classical determinant theory.

At this stage the application of the new theory culminates in the following theorem (see [14]). Let $R$ be a Lie nilpotent algebra (over $\mathbb{Q}$ ) of index $k \geq 1$. If $\delta: R \longrightarrow R$ is an automorphism with $\delta^{n}=\mathrm{id}_{R}$, then the skew polynomial algebra $R[w, \delta]$ is right 
integral over $\operatorname{Fix}(\delta)\left[w^{n}\right]$ of degree $n^{k}$. In other words, for any $f(w) \in R[w, \delta]$ we have

$$
g_{0}\left(w^{n}\right)+f(w) g_{1}\left(w^{n}\right)+\cdots+f^{n^{k}-1}(w) g_{n^{k}-1}\left(w^{n}\right)+f^{n^{k}}(w)=0
$$

for some $g_{t}\left(w^{n}\right) \in \operatorname{Fix}(\delta)\left[w^{n}\right], 0 \leq t \leq n^{k}-1$.

In case of an arbitrary $R$ and $A \in \mathrm{M}_{n}(R)$, we have

$$
n\left(x I_{n}-A\right)\left(x I_{n}-A\right)^{*}=p_{A, 1}(x) I_{n}+C_{0}+C_{1} x+\cdots+C_{n} x^{n},
$$

where the matrices $C_{i} \in \mathrm{M}_{n}(R)$ are uniquely determined by $A, \operatorname{tr}\left(C_{i}\right)=0$ and each entry of $C_{i}$ is in the additive subgroup $[R, R]$ generated by the commutators, i.e. $C_{i} \in \mathrm{M}_{n}([R, R])$ for all $0 \leq i \leq n$. Now the identity

$\left(\lambda_{0}^{(1)} I_{n}+C_{0}\right)+A\left(\lambda_{1}^{(1)} I_{n}+C_{1}\right)+\cdots+A^{n-1}\left(\lambda_{n-1}^{(1)} I_{n}+C_{n-1}\right)+A^{n}\left(n ! I_{n}+C_{n}\right)=0$ with right matrix coefficients holds for $A$. Since commutativity of $R$ would imply $C_{0}=C_{1}=\cdots=C_{n-1}=C_{n}=0$, this is a direct right sided generalization of the classical Cayley-Hamilton theorem.

All the results above have a natural left sided version.

Matrix algebras over the infinitely generated Grassmann algebra, which is Lie nilpotent of index 2, are particularly in the purview of this non-commutative determinant theory. Polynomial identities satisfied by these matrix algebras play a central role in Kemer's classification of T-prime T-ideals.

\section{CEnTralizers AND ZeRo LEVEl CENTRALIZERS}

For a linear transformation of a finite dimensional vector space over an algebraically closed field the existence of the Jordan normal base is well known in elementary linear algebra. An astonishing fact is that a far reaching generalization of this fundamental result can be formulated for nilpotent complete join homomorphisms of lattices (see [13]). The description of the centralizers and zero level centralizers (two sided annihilators) in Szigeti's papers $([1,17])$ depends on the use of the following remarkable consequence of this lattice theoretical Jordan normal base theorem.

A doubly indexed subset $X=\left\{x_{\gamma, i} \mid \gamma \in \Gamma, 1 \leq i \leq k_{\gamma}\right\}$ of a (unitary) left $R$ module ${ }_{R} M$ is called a nilpotent Jordan normal base with respect to $\varphi \in \operatorname{End}_{R}(M)$ if each $R$-submodule $R x_{\gamma, i} \leq M$ is simple,

$$
\underset{\gamma \in \Gamma, 1 \leq i \leq k_{\gamma}}{\oplus} R x_{\gamma, i}=M
$$

is a direct sum, $\varphi\left(x_{\gamma, i}\right)=x_{\gamma, i+1}$, for all $\gamma \in \Gamma, 1 \leq i \leq k_{\gamma}-1, \varphi\left(x_{\gamma, k_{\gamma}}\right)=0$ and the set $\left\{k_{\gamma} \mid \gamma \in \Gamma\right\}$ of integers is bounded ( $\Gamma$ is called the set of Jordan blocks and the size of the block $\gamma \in \Gamma$ is the integer $k_{\gamma}$ ). The existence of such Jordan normal base implies that $\varphi^{n}=0 \neq \varphi^{n-1}$, where $n=\max \left\{k_{\gamma} \mid \gamma \in \Gamma\right\}$. 
For $\varphi \in \operatorname{End}_{R}(M)$ the following are equivalent:

(i) ${ }_{R} M$ is semisimple and $\varphi$ is nilpotent.

(ii) ${ }_{R} M$ has a nilpotent Jordan normal base with respect to $\varphi$.

The rest of the section follows literally the exposition in [1] and [17].

If $R$ is a local ring with Jacobson radical $J$ and ${ }_{R} M$ is a finitely generated semisimple left $R$-module, then the centralizer $\operatorname{Cen}(\varphi)$ of a nilpotent $\varphi \in \operatorname{End}_{R}(M)$ is isomorphic to the opposite of the factor $\mathcal{N}(X) / \mathcal{l}(X)$ as an algebra over the center $\mathrm{Z}(R)$ :

$$
\operatorname{Cen}(\varphi)=\left\{\psi \in \operatorname{End}_{R}(M) \mid \psi \circ \varphi=\varphi \circ \psi\right\} \cong(\mathcal{N}(X) / \mathcal{d}(X))^{\mathrm{op}},
$$

where

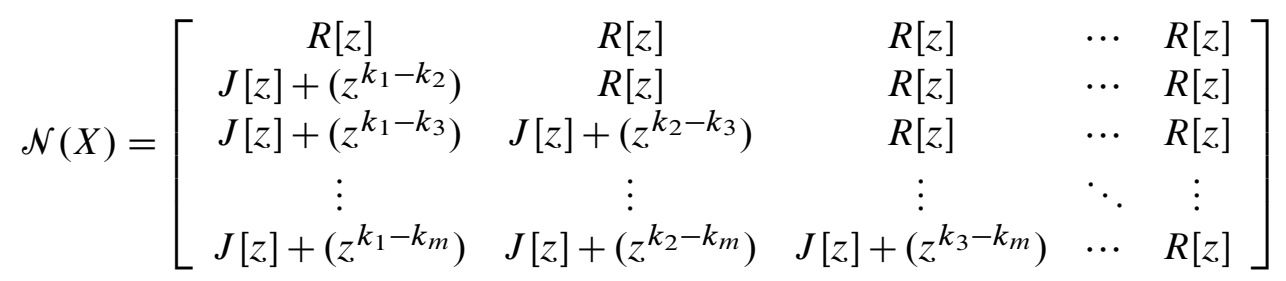

is a subalgebra of $\mathrm{M}_{m}(R[z])$ and

$$
d(X)=\left[\begin{array}{cccc}
J[z]+\left(z^{k_{1}}\right) & J[z]+\left(z^{k_{2}}\right) & \cdots & J[z]+\left(z^{k_{m}}\right) \\
J[z]+\left(z^{k_{1}}\right) & J[z]+\left(z^{k_{2}}\right) & \cdots & J[z]+\left(z^{k_{m}}\right) \\
\vdots & \vdots & \ddots & \vdots \\
J[z]+\left(z^{k_{1}}\right) & J[z]+\left(z^{k_{2}}\right) & \cdots & J[z]+\left(z^{k_{m}}\right)
\end{array}\right]
$$

is an ideal of $\mathcal{N}(X)$. The number of blocks $m=\operatorname{dim}_{R}(\operatorname{ker}(\varphi)), \Gamma=\{1,2, \ldots, m\}$ and the finite sequence of block sizes $k_{1} \geq k_{2} \geq \ldots \geq k_{m} \geq 1$ in a nilpotent Jordan normal base $X=\left\{x_{\gamma, i} \mid \gamma \in \Gamma, 1 \leq i \leq k_{\gamma}\right\}$ with respect to $\varphi$ are independent of the choice of $X$ and uniquely determined by $\varphi$.

For another not necessarily nilpotent endomorphism $\sigma \in \operatorname{End}_{R}(M)$, the containment $\operatorname{Cen}(\varphi) \subseteq \operatorname{Cen}(\sigma)$ holds if and only if there is an $R$-generating set $\left\{y_{j} \in M \mid\right.$ $1 \leq j \leq d\}$ of ${ }_{R} M$ and there are elements $a_{1}, a_{2}, \ldots, a_{n} \in R$ such that

$$
a_{1} \psi\left(y_{j}\right)+a_{2} \varphi\left(\psi\left(y_{j}\right)\right)+\cdots+a_{n} \varphi^{n-1}\left(\psi\left(y_{j}\right)\right)=\sigma\left(\psi\left(y_{j}\right)\right)
$$

for all $1 \leq j \leq d$ and all $\psi \in \operatorname{Cen}(\varphi)$.

Since $\operatorname{Cen}(\varphi) \subseteq \operatorname{Cen}(\sigma)$ is equivalent to $\sigma \in \operatorname{Cen}(\operatorname{Cen}(\varphi))$, the last statement can be viewed as a non-commutative generalization of Schur's double centralizer theorem in the nilpotent case.

Keeping the above conditions and notations, the zero-level centralizer $\operatorname{Cen}_{0}(\varphi)$ of the nilpotent $\varphi$ is isomorphic to the opposite of the factor $\mathcal{N}_{0}(X) / \mathcal{d}(X)$ as a $\mathrm{Z}(R)$ algebra:

$$
\operatorname{Cen}_{0}(\varphi)=\left\{\psi \in \operatorname{End}_{R}(M) \mid \psi \circ \varphi=\varphi \circ \psi=0\right\} \cong\left(\mathcal{N}_{0}(X) / d(X)\right)^{\mathrm{op}},
$$


where

$$
\mathcal{N}_{0}(X)=\left[\begin{array}{cccc}
J[z]+\left(z^{k_{1}-1}\right) & J[z]+\left(z^{k_{2}-1}\right) & \cdots & J[z]+\left(z^{k_{m}-1}\right) \\
J[z]+\left(z^{k_{1}-1}\right) & J[z]+\left(z^{k_{2}-1}\right) & \cdots & J[z]+\left(z^{k_{m}-1}\right) \\
\vdots & \vdots & \ddots & \vdots \\
J[z]+\left(z^{k_{1}-1}\right) & J[z]+\left(z^{k_{2}-1}\right) & \cdots & J[z]+\left(z^{k_{m}-1}\right)
\end{array}\right]
$$

is a subalgebra of $\mathrm{M}_{m}(R[z])$, still containing $d(X)$ as an ideal.

Now let $\varphi \in \operatorname{End}_{R}(M)$ be an arbitrary $R$-endomorphism of a finitely generated semisimple left $R$-module ${ }_{R} M$. Then there exist $R$-submodules $W_{1}, W_{2}$ and $V$ of $M$ such that $W=W_{1} \oplus W_{2}$ and $M=V \oplus W$ are direct sums, $\operatorname{ker}(\varphi) \subseteq W, \varphi(W)=W_{2}$, $\varphi(V)=V, \operatorname{dim}_{R}\left(W_{1}\right)=\operatorname{dim}_{R}(\operatorname{ker}(\varphi)),(\varphi \uparrow W) \in \operatorname{End}_{R}(W)$ is nilpotent and for the zero-level centralizer of $\varphi$ we have $\operatorname{Cen}_{0}(\varphi) \cong T$, where

$$
T=\left\{\theta \in \operatorname{End}_{R}(W) \mid \theta\left(W_{1}\right) \subseteq \operatorname{ker}(\varphi) \text { and } \theta\left(W_{2}\right)=\{0\}\right\}=\operatorname{Cen}_{0}(\varphi \uparrow W) .
$$

The double zero-centralizer theorem states that for another endomorphism $\sigma \in \operatorname{End}_{R}(M)$ the following conditions are equivalent:

(i) $\sigma \in \mathrm{Cen}_{0}\left(\operatorname{Cen}_{0}(\varphi)\right)$,

(ii) $\operatorname{Cen}_{0}(\varphi) \subseteq \operatorname{Cen}_{0}(\sigma)$,

(iii) $\operatorname{ker}(\varphi) \subseteq \operatorname{ker}(\sigma)$ and $\operatorname{im}(\sigma) \subseteq \operatorname{im}(\varphi)$.

\section{REFERENCES}

[1] V. Drensky, J. Szigeti, and L. van Wyk, "Centralizers in endomorphism rings," J. Algebra, vol. 324, no. 12, pp. 3378-3387, 2010.

[2] P. Körtesi and J. Szigeti, "On permanental identities over matrix rings," Commun. Algebra, vol. 22, no. 1, pp. 159-171, 1994.

[3] P. Körtesi and J. Szigeti, "The adjacency matrix of a directed graph over the Grassmann algebra," in Algebra and its applications. Proceedings of the international conference, Athens, OH, USA, March 25-28, 1999. Providence, RI: American Mathematical Society (AMS), 2000, pp. 319321.

[4] A. Lee, G. Révész, J. Szigeti, and Z. Tuza, "Capelli polynomials, almost-permutation matrices and sparse Eulerian graphs,” Discrete Math., vol. 230, no. 1-3, pp. 49-61, 2001.

[5] J. Meyer, J. Szigeti, and L. van Wyk, "A Cayley-Hamilton trace identity for $2 \times 2$ matrices over Lie-solvable rings," Linear Algebra Appl., vol. 436, no. 7, pp. 2578-2582, 2012.

[6] S. Sehgal and J. Szigeti, "Matrices over centrally $\mathbb{Z}_{2}$-graded rings," Beitr. Algebra Geom., vol. 43, no. 2, pp. 399-406, 2002.

[7] J. Szigeti, "Permanental polynomial identities on matrix rings," J. Algebra, vol. 165, no. 2, pp. 389-393, 1994.

[8] J. Szigeti, "Eulerian *-polynomial identities on matrix algebras," Commun. Algebra, vol. 23, no. 1, pp. 245-253, 1995.

[9] J. Szigeti, "New determinants and the Cayley-Hamilton theorem for matrices over Lie nilpotent rings," Proc. Am. Math. Soc., vol. 125, no. 8, pp. 2245-2254, 1997.

[10] J. Szigeti, "Idempotent ideals in Lie nilpotent rings," in Methods in ring theory. Proceedings of the Trento conference, Trento, Italy. New York, NY: Marcel Dekker, 1998, pp. 287-292.

[11] J. Szigeti, "On the characteristic polynomial of supermatrices," Isr. J. Math., vol. 107, pp. 229$235,1998$. 
[12] J. Szigeti, "Cayley-Hamilton theorem for matrices over an arbitrary ring," Serdica Math. J., vol. 32, no. 2-3, pp. 269-276, 2006.

[13] J. Szigeti, "Linear algebra in lattices and nilpotent endomorphisms of semisimple modules," $J$. Algebra, vol. 319, no. 1, pp. 296-308, 2008.

[14] J. Szigeti, "A new class of matrix algebras," arXiv:1501.07104, 2015.

[15] J. Szigeti and Z. Tuza, "Solving systems of linear equations over Lie nilpotent rings," Linear Multilinear Algebra, vol. 42, no. 1, pp. 43-51, 1997.

[16] J. Szigeti, Z. Tuza, and G. Révész, "Eulerian polynomial identities on matrix rings," J. Algebra, vol. 161, no. 1, pp. 90-101, 1993.

[17] J. Szigeti and L. van Wyk, "The zero-level centralizer in endomorphism algebras," Proc. R. Soc. Edinb., Sect. A, Math., vol. 142, no. 6, pp. 1325-1336, 2012.

[18] J. Szigeti and L. van Wyk, "Determinants for $n \times n$ matrices and the symmetric Newton formula in the $3 \times 3$ case," Linear Multilinear Algebra, vol. 62, no. 8, pp. 1076-1090, 2014.

[19] J. Szigeti and L. van Wyk, "On Lie nilpotent rings and Cohen's Theorem," Communications in Algebra, vol. 43, no. 11, pp. 4783-4796, 2015.

\section{Author's address}

\section{Stephan Foldes}

Department of Mathematics, Tampere University of Technology, Tampere, Finland 